\title{
ANÁLISE ECONÔMICA DE SISTEMAS AGROFLORESTAIS VIA USO DE EQUAÇÕES DIFERENCIAIS ${ }^{1}$
}

\author{
Juliana Galvão de Sousa Magalhães², Márcio Lopes da Silva³, Thiago Taglialegna Salles² e Lyvia \\ Julienne Sousa Rego ${ }^{2}$
}

\begin{abstract}
RESUMO - A aplicação de diferentes técnicas de análise econômica em sistemas agroflorestais é de grande importância para suporte às tomadas de decisões. Assim, testou-se um modelo de equação diferencial como metodologia de análise da viabilidade econômica de um sistema agroflorestal (SAF), observando se havia influência das receitas intermediárias de uma cultura agrícola no período de rotação da cultura arbórea, em dois horizontes de planejamento: um único corte e vários cortes, executando-se a reforma ou a substituição após o corte do alto fuste. Concluiu-se que, para horizonte de planejamento de um corte, variações na receita da cultura agrícola não afetarão a idade ótima de corte. Entretanto, variações na receita agrícola implicarão em variações inversamente proporcionais na idade ótima de corte quando se planeja um horizonte com vários cortes das árvores, ou seja, devido ao lucro com o cultivo agrícola, é economicamente viável antecipar o corte da madeira e realizar a reforma do plantio.
\end{abstract}

Palavras-chave: Economia florestal; Rotação florestal; Agrossilvicultura.

\section{ECONOMIC ANALYSIS OF THE AGROFORESTRY SYSTEMS BY USING DIFFERENTIAL EQUATIONS}

\begin{abstract}
The use of economic analysis techniques on agroforestry systems is important to support decisionmakers. Therefore, we tested a differential equation model to analyze the economic viability of an agroforestry system (AFS). Thus, the objective of the present study was to verify the occurrence of is any influence of the intermediary income in an agricultural crop in the rotation period of the tree crop in two planning scenarios: a single cut and several cut, which provide the renovation or replacement after the cutting of tall trees. We concluded that, for a planning scenario of one cut, variations in crop revenues will not affect the rotation age. However, variations in farm revenue will lead to inversely proportional variations on the rotation age when a planning scenario with several cuts is implemented. In other words, due to the profit from agricultural cultivation, it is economically feasible to anticipate the cutting of wood and carry out the reform of planting.
\end{abstract}

Keywords: Forest economy; Rotation Forestry; Agroforestry.

\section{INTRODUÇÃO}

Os Sistemas Agroflorestais (SAFs), pela aproximação com os ecossistemas naturais em estrutura e diversidade, representam grande potencial para a restauração de ambientes degradados. Além disso, apresentam papel relevante como alternativa de produção, pois permitem equilibrar a oferta de produtos agrícolas e florestais com a prestação de serviços ambientais (PASSOS; COUTO, 1997).
Os SAFs podem ser empregados tanto como estratégia de restauração, ou constituição de agroecossistemas sustentáveis, quanto para valorização da qualidade dos serviços ambientais (CAMPELLO et al., 2006). Ademais, oferecem simultaneamente uma variedade de produtos florestais e não florestais, proporcionando ao agricultor maior flexibilidade na comercialização de seus produtos. Diversificar a produção permite também um fluxo de caixa mais regular e uma

\footnotetext{
${ }^{1}$ Recebido em 20.06.2013 aceito para publicação em 03.12.2013.

${ }^{2}$ Programa de Pós-Graduação em Ciencias Florestais, Departamento de Engenharia Florestal, Universidade Federal de Viçosa. E-mail: <jgsmagalhaes@gmail.com>,<thiagotsalles@gmail.com>e <lyviajulienne@hotmail.com>.

${ }^{3}$ Departamento de Engenharia Florestal da Universidade Federal de Viçosa, UFV, Brasil. E-mail: <marlosil@ufv.br>.
} 
racionalização da mão de obra (SANTOS; PAIVA, 2002), o que, consequentemente, reduzirá os custos de produção (DOSSA, 2000).

Os SAFs são classificados de acordo com os tipos de componentes incluídos, como estrutura espacial e arranjo temporal (OTS/CATIE, 1986). Nos sistemas agroflorestais denominados sequenciais, os cultivos agrícolas anuais e as plantações de árvores se sucedem no tempo. Por exemplo, os sistemas de agricultura migratória, sistema silviagrícola rotativo (capoeiras melhoradas com espécies arbóreas de rápido crescimento) e sistema Taungya (cultivos anuais consorciados apenas durante os primeiros anos de estabelecimento de árvores, sendo seu objetivo principal a produção de madeira).

Os sistemas agroflorestais classificados como simultâneos integram, ao mesmo tempo, os cultivos anuais e perenes, com espécies madeireiras ou de uso múltiplo e, ou, pecuária. Já os chamados sistemas complementares, como as cercas vivas e cortinas quebravento, podem estar associados aos sistemas sequenciais ou simultâneos. Essa classificação descritiva é mais didática, pois o nome de cada sistema indica seus principais componentes, permitindo, assim, uma ideia de sua fisionomia, principais funções e objetivos (ENGEL, 1999).

A prática desses sistemas é extremamente positiva para a sustentabilidade do ambiente. No entanto, são necessários estudos complementares que foquem outros aspectos importantes e promovam melhor aproveitamento do seu potencial. Entre esses está a incorporação das análises econômicas e financeiras, as quais avaliam os SAFs quanto à sua viabilidade econômica e rotação florestal e, assim, motivem sua implementação no setor florestal e nos sistemas de produção brasileiros.

O teste de análise econômica consiste em verificar se as receitas inerentes ao empreendimento superam os custos necessários. A aplicação dos critérios de análise econômica na área florestal é fundamental como ferramenta de tomada de decisão; assim, é possível decidir qual o melhor projeto ou a melhor alternativa de manejo a ser adotada.

Determinar a idade econômica de corte, o espaçamento, a adubação, a época e a intensidade de tratamentos silviculturais, entre outras decisões, podem ser tomadas de forma mais segura quando executadas as simulações baseadas nos critérios técnico-econômicos (LOPES, 1990). Todo projeto antes de ser implementado

Revista Árvore, Viçosa-MG, v.38, n.1, p.73-79, 2014 deve submeter-se aos testes de viabilidade econômica. Além disso, é importante proceder às análises técnica, social, política e ambiental.

A maioria dos estudos utiliza os critérios conhecidos como Valor Presente Líquido (VPL) e Benefício Periódico Equivalente (BPE), para avaliação da rotação econômica e estudos de viabilidade econômica em projetos de SAF (RODIGHERI, 1997; DUBÈ et al., 2000; SANTOS et al., 2000; SANTOS; PAIVA, 2002; RIBEIRO et al., 2004, 2007; SOUZA et al., 2007). Ambos os critérios levam em conta a variação do capital no tempo, ou seja, atribuem diferentes ponderações às receitas líquidas em função de sua distribuição ao longo do tempo (REZENDE; OLIVEIRA, 1995). Entretanto, cada um aponta diferentes aspectos relacionados aos projetos (SILVA; FONTES, 2005).

O Valor Presente Líquido (VPL) de um projeto de investimento é definido como a soma algébrica dos valores descontados do fluxo de caixa a ele associado, ou seja, é a diferença entre o valor presente das receitas e o valor presente dos custos, após definida a taxa de desconto (i) (SILVA; FONTES, 2005). O projeto que apresentar o VPL positivo é economicamente viável, sendo considerado o melhor aquele que apresentar maior VPL.

O Benefício Periódico Equivalente (BPE) ou Valor Anual Equivalente (VAE) é indicado na seleção de projetos que apresentam durações ou vidas úteis diferentes, pois esse critério converte os fluxos de caixa líquidos em uma série equivalente de valores iguais e, geralmente, anuais (REZENDE; OLIVEIRA, 1995). Dito de outra forma, o VAE transforma o valor atual do projeto, ou o seu VPL, em fluxo de receitas ou custos periódicos e contínuos, equivalentes ao valor atual, durante a vida útil do projeto (SILVA; FONTES, 2005).

Outro método matemático que pode ser aplicado à economia é o cálculo diferencial (MARQUES et al., 2005), o qual se dedica ao estudo de derivada, ou seja, da variação, do movimento, ou melhor, do deslocamento de um gráfico. A vantagem desse critério é a possibilidade de determinar o ótimo econômico, o ponto que maximiza o lucro ou minimiza os custos.

No estudo realizado por Lopes (1990), aplicou-se o cálculo diferencial no Valor Atual dos itens de custos e receitas para determinação do ótimo econômico, ou 
seja, a idade ótima de corte, que é considerada como o ponto do tempo em que ocorre a maximização dos lucros ou a minimização dos custos. As aplicações de técnicas de modelagem em sistemas agroflorestais apresentam-se como ferramenta eficiente e confiável para dar suporte às tomadas de decisões.

Diante do exposto, este estudo apresenta como objetivo o teste de um modelo de equação diferencial como metodologia de análise da viabilidade econômica de um SAF. Pretendeu-se, ainda, observar a influência de receitas intermediárias de uma cultura agrícola no período de rotação da cultura arbórea, em dois horizontes de planejamento: um único corte e vários cortes, efetuando a reforma ou a substituição após o corte do alto fuste.

\section{MATERIAL E MÉTODOS}

\subsection{Caracterização do Sistema Agroflorestal (SAF)}

O modelo hipotético considerado foi um sistema Taungya de consórcio entre a espécie agrícola feijão (Phaseolus vulgaris) e a cultura arbórea eucalipto (Eucalyptus sp.) plantada em espaçamento de $3 \mathrm{x}$ $2 \mathrm{~m}$. O feijão foi implantado entre duas linhas de eucalipto no primeiro ano de ocorrência do sistema, com área de $18 \mathrm{~m}^{2}$, resultando em uma densidade de 200.000 plantas por hectare. Os custos envolvidos na instalação e as receitas do projeto são descritos na Tabela 1.

O modelo de produção volumétrica utilizado expressa a produção de madeira por hectare em função da idade (BINOTI, 2010). Utilizou-se apenas o fator idade como variável independente, pois este fornece boa estimativa final, além de explicar bem a produção obtida (equação 1).

$$
V\left(m^{3} / h a\right)=\frac{485.68}{1+7.4275 *\left[\exp ^{-0.4062 * I(\text { anos })}\right]}
$$

O método de avaliação econômica utilizado no estudo foi o Valor Presente Líquido (VPL), o qual permite calcular o lucro corrigido para o início do projeto. Assim, os rendimentos obtidos em diferentes pontos no tempo tornam-se mensuráveis devido à transformação em seus equivalentes valores atuais. É um método muito utilizado na avaliação de investimentos florestais, pois considera a variação do capital no tempo (SILVA et al., 2002).

$$
\begin{aligned}
& V P L=\sum_{j} \text { Receitas }-\sum_{j=0} \text { Custos } \\
& V P L=\sum_{j}^{n} \mathrm{R}_{j}(1+i)^{-1}-\sum_{j=0}^{n} \mathrm{C}_{j}(1+i)^{-1}
\end{aligned}
$$

em que $R_{j}=$ valor atual das receitas; $C_{j}=$ valor atual dos custos; $\mathrm{i}$ = taxa de juros; $\mathrm{j}$ = período em que a receita ou o custo ocorrem; e n = número máximo de períodos.

A obtenção da idade ótima de corte foi possível pelo uso das derivadas de primeira ordem $\left(\partial^{\prime}=0\right)$, também denominada como condição necessária, e as de segunda ordem ( $\partial$ "'>0 ou $\partial$ "'<0), as quais são ditas condições suficientes. O sinal negativo ou positivo da derivada de segunda ordem indicou a ocorência de um máximo ou mínimo da função.

Consideraram-se dois horizontes de planejamento: um de único corte (uma rotação) e outro de vários cortes, efetuando-se a reforma ou a substituição após o corte do alto fuste.

\section{2. Horizonte de planejamento de um corte}

Neste caso, tem-se um período “ $T$ ” de produção, ou seja, o modelo se restringe a um investimento isolado, cujo Valor Presente Líquido (VPL) pode ser representado pela equação (3), conforme demonstrado por Lopes (1990):

$$
\begin{aligned}
& V P L=\frac{p V-C B-c V}{e^{r T}+\frac{R C}{e^{r}}}- \\
& \mathrm{K}-\frac{A T+C C P *\left(e^{r T}-1\right)}{r * e^{r T}}-\frac{C C 1 * e^{r}+C C 2}{e^{2 r}}
\end{aligned}
$$

Considera-se como idade ótima de corte o valor de “T”, que maximize a expressão. Logo, a condição necessária, ou de primeira ordem, de otimização de duração deste investimento é dada por: $\frac{\partial V P L}{\partial T}=0$. Para a condição de segunda ordem, tem-se que: $\frac{\partial^{2} V P L}{\partial T^{2}}<0$.

\section{3. Horizonte de planejamento de vários cortes}

Para esse horizonte de planejamento, considerou-se que o projeto será replicado, ou seja, será efetuada reforma ou substituição após o corte do alto fuste. O modelo é constituído por uma cadeia infinita de investimentos. Neste caso, o valor atual da cadeia infinita é denominado custo de oportunidade da terra (B).

Revista Árvore, Viçosa-MG, v.38, n.1, p.73-79, 2014 


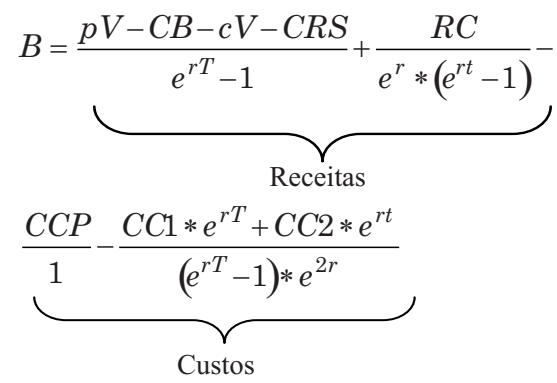

*As variáveis descritas nas equações (3) e (4) foram definidas na Tabela 1.

O custo de implantação não foi considerado nesta expressão, pois ele é de interesse somente quando se estuda a dimensão do empreendimento (LOPES, 1990).

\subsection{Análise estática comparativa}

A estática comparativa é uma técnica matemática pela qual se investiga um modelo econômico, comparando uma condição estática (de equilíbrio) com outra. É uma técnica analítica primária muito utilizada no estudo de mercado, em que se pode analisar como o preço e a quantidade no equilíbrio são afetados por mudanças na demanda e oferta (SILBERBERG; SUEN, 2000).

Neste estudo, a técnica foi utilizada para avaliar os dois diferentes resultados econômicos, antes e depois de implantada a cultura arbórea.

\section{RESULTADOS}

\subsection{Fluxo de caixa}

De posse da planilha de custos, elaborou-se o fluxo de caixa do projeto. Ressalta-se que no ano de colheita da madeira foram considerados também a receita com a venda ( $\left.\mathrm{p}^{*} \mathrm{~V}\right)$ e o custo dessa colheita [182,19 $+(20 * \mathrm{~V})]$.

\subsection{Horizonte de planejamento de um único corte}

Derivando o modelo do VPL (equação 3) em função do tempo, obtém-se:

$$
\begin{aligned}
& \frac{\partial V P L}{\partial T}=\frac{1}{e^{r t}} *\left(p \frac{\partial V}{\partial T}-\mathrm{c} \frac{\partial V}{\partial T}-r p V+\right. \\
& r C B+r c V-A T-C C P)=0
\end{aligned}
$$

Tal função também pode ser escrita como:

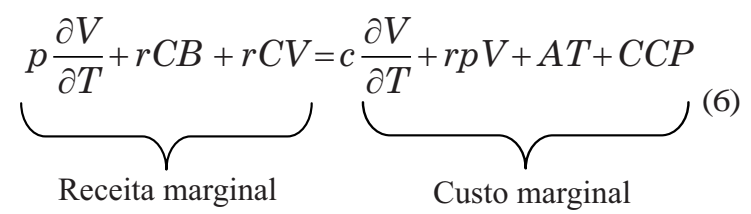

Ao efetuar a análise estática comparativa, observou-se que: $\frac{\partial T}{\partial R C}=0$, ou seja, a receita da cultura agrícola não interferiu na duração da idade ótima de corte.

Tabela 1 - Itens de custos e receitas envolvidos na implantação de um sistema agroflorestal.

\begin{tabular}{|c|c|c|c|}
\hline Códigos & Itens & Valores & Unidades \\
\hline $\mathrm{p}$ & Preço da madeira & 64 & $\mathrm{R} \$ / \mathrm{m}^{3}$ \\
\hline $\mathrm{r}$ & Taxa de desconto & 0,08 & ao ano \\
\hline VT & Valor da terra & 3600 & $\mathrm{R} \$ / \mathrm{ha}$ \\
\hline $\mathrm{AT}$ & Aluguel da terra $=\mathrm{vt} \times \mathrm{r}$ & 288 & $\mathrm{R} \$ /$ ha \\
\hline $\mathrm{C}$ & Custo de colheita & 20 & $\mathrm{R} \$ / \mathrm{m}^{3}$ \\
\hline CRS & Reforma & 2.841 & $\mathrm{R} \$ /$ ha \\
\hline K & Implantação & 4059,05 & $\mathrm{R} \$ /$ ha \\
\hline CCP & Manutenção anual & 212,19 & $\mathrm{R} \$ / \mathrm{ha}$ \\
\hline CC1 & Tratos culturais $1^{\circ}$ ano & 800 & $\mathrm{R} \$ / \mathrm{ha}$ \\
\hline CC2 & Tratos culturais $2^{\circ}$ ano & 600 & $\mathrm{R} \$ /$ ha \\
\hline $\mathrm{CB}$ & Bateção pré-corte & 182,19 & $\mathrm{R} \$ / \mathrm{ha}$ \\
\hline \multirow[t]{3}{*}{ FeijãoRC (receita) } & Produtividade & 600 & $\mathrm{~kg} / \mathrm{ha}$ ano \\
\hline & Custo colheita & 190,5 & $\mathrm{R} \$ /$ ha ano \\
\hline & Preço da saca de $60 \mathrm{~kg}$ & 150,180 e $240 *$ & $\mathrm{R} \$ /$ saca \\
\hline
\end{tabular}
Table 1 - Costs and revenues involved on the implementation of an agroforestry system.

* Fonte: <http://www.noticiasagricolas.com.br/cotacoes/graos>.

Revista Árvore, Viçosa-MG, v.38, n.1, p.73-79, 2014 
Observou-se que, pelo método do VPL, a idade ótima de corte do povoamento ocorre aos 7 anos; já pelo critério de equivalência entre receita marginal e custo marginal (análise gráfica), a idade ótima de corte pode ser considerada aos 8 anos (Figura 1).

\subsection{Horizonte de planejamento de infinitos cortes}

Derivando o modelo do VPL (equação 4) em função do tempo, obtém-se:

$$
\begin{aligned}
& \frac{\partial B}{\partial T}=\frac{1}{\left(e^{r T}-1\right)} *\left(\frac{\partial V}{\partial T}-c \frac{\partial V}{\partial T}-r p V+\right. \\
& r C B+r c V+r C R S-r B-C C P)=0
\end{aligned}
$$

Tal função também pode ser escrita como:

$$
\underbrace{p \frac{\partial V}{\partial T}+r C B+r c V+r C R S}_{\text {Receita marginal }}=c \underbrace{c \frac{\partial V}{\partial T}+r p V+r B+C C P}_{\text {Custo marginal }}
$$

Na análise estática comparativa, observou-se que a variação na idade ótima de corte em função da receita agrícola (RC) é um valor menor que zero, ou seja, a duração da idade de corte apresentou reduções à medida que a receita da cultura agrícola aumenta:

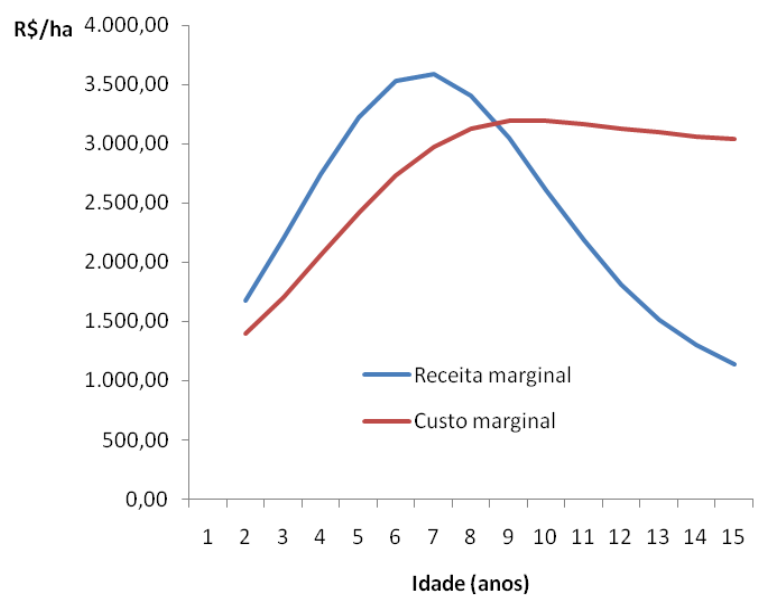

Figura 1 - Idade ótima de corte estipulada pelo método gráfico de equivalência entre a receita marginal e o custo marginal, considerando-se o horizonte de planejamento de um único corte.

Figure 1 - Optimal cutting age stipulated by the graphical method of equivalence between marginal revenue and marginal cost, considering a planning horizon of a single cut.

$$
\frac{\partial T}{\partial R C}=\frac{r /\left[e^{r}\left(e^{r t}-1\right)\right]}{p \frac{\partial^{2} V}{\partial T^{2}}+r c \frac{\partial V}{\partial T}-c \frac{\partial^{2} V}{\partial T^{2}}-r p \frac{\partial V}{\partial T}}<0
$$

Nesta situação de planejamento, também se observou variação entre os valores de idade ótima determinada pelo critério do VPL e pelo método gráfico (Figura 2).

Variando o preço de venda da saca de feijão de $\mathrm{R} \$ 150$ para $\mathrm{R} \$ 180$, verificou-se que o VPL aumentou de $\mathrm{R} \$ 4.776,33$ para $\mathrm{R} \$ 5.496,6$ e que houve redução da idade ótima de corte (Figura 3).

Considerando-se o preço de venda do feijão como R\$240/saca, o VPL aumentou para R\$7.121,49. Já a idade ótima de corte da cultura arbórea reduziu para, aproximadamente, 8 anos (Figura 4).

\section{DISCUSSÃO}

\subsection{Horizonte de planejamento de um corte}

A análise estática comparativa foi efetiva para comparar os diferentes estados de equilíbrio que se associaram aos diferentes conjuntos de valores e variáveis que determinam a idade ótima de corte. Nessa situação, observou-se que: $\frac{\partial T}{\partial R C}=0$. Tal situação indica que, considerando um horizonte de planejamento de um corte, variações nos rendimentos do consórcio com a cultura agrícola (RC) no primeiro ano de implantação do sistema não afetarão a idade ótima de corte da cultura arbórea.

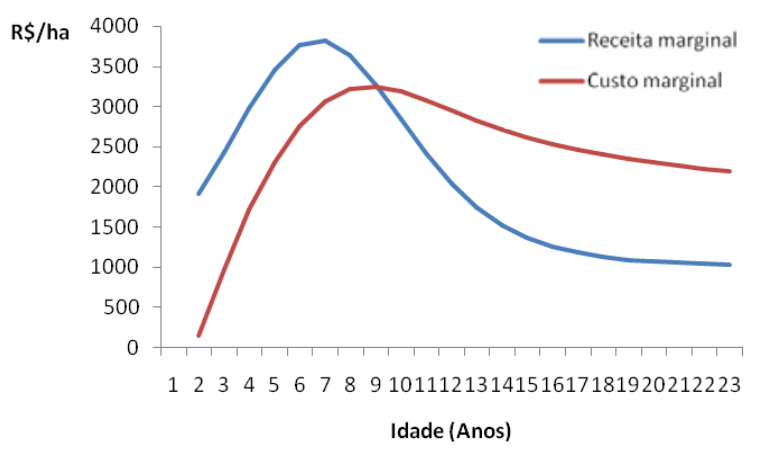

Figura 2 - Idade ótima de corte estipulada pelo método gráfico, considerando-se o preço de venda da cultura agrícola de $\mathrm{R} \$ 150 /$ saca.

Figure 2 - Optimal cutting age stipulated by the graphical method, considering the selling price of the crop at $R \$ 150 /$ bag.

Revista Árvore, Viçosa-MG, v.38, n.1, p.73-79, 2014 


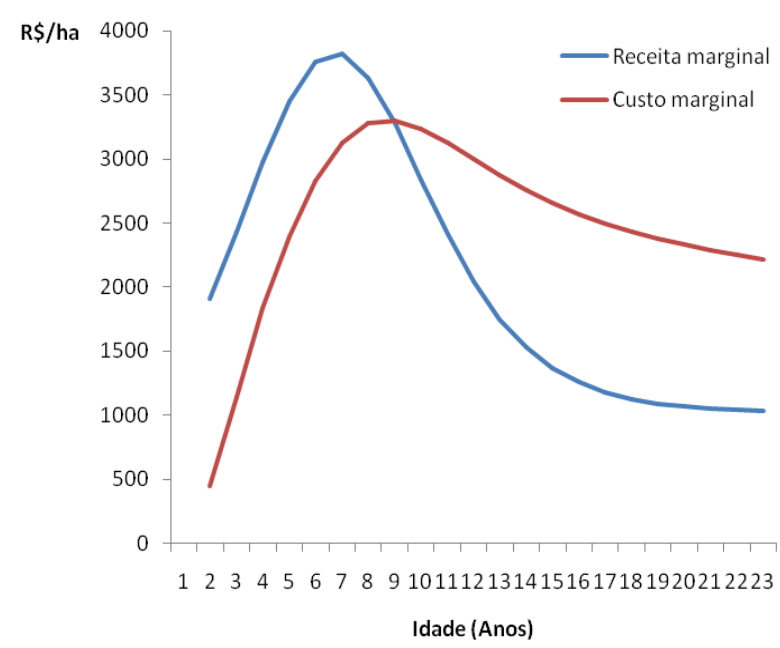

Figura 3 - Idade ótima de corte estipulada pelo método gráfico, considerando-se o preço de venda da cultura agrícola de $\mathrm{R} \$ 180 /$ saca.

Figure 3 - Optimal cutting age stipulated by the graphical method, considering the selling price of the crop at $R \$ 180 / \mathrm{bag}$.

A diferença entre as idades ótimas de corte estimadas pelos dois métodos (VPL e análise gráfica) ocorreu devido às características de cada processo: no critério de VPL, a análise se restringe a intervalos periódicos anuais, ou seja, esse método sempre fornecerá resultados inteiros, enquanto o método de análise gráfica já abrange um intervalo contínuo, permitindo resultados mensais.

Diante disso, considerando o horizonte de planejamento de um corte, o ótimo econômico para a cultura arbórea pode ser obtido ao final do sétimo até o início do oitavo ano de implantação do sistema agroflorestal, como evidenciado na Figura 1.

\subsection{Horizonte de planejamento de vários cortes}

Pelo fato de $\frac{\partial T}{\partial R C}<Q$ variações proporcionais na receita agrícola implicam em variações inversamente proporcionais na idade ótima de corte, ou seja, se aumentar o valor pago pela saca do feijão, consequentemente essas receitas intermediárias aumentarão o VPL. Assim, torna-se economicamente interessante antecipar a idade de corte da cultura arbórea, executar a reforma ou a substituição e reimplantar a cultura agrícola.

No horizonte de vários cortes, o aumento no valor da saca de $\mathrm{R} \$ 150$ para $\mathrm{R} \$ 240$ proporcionou variação positva no VPL, possibilitando também

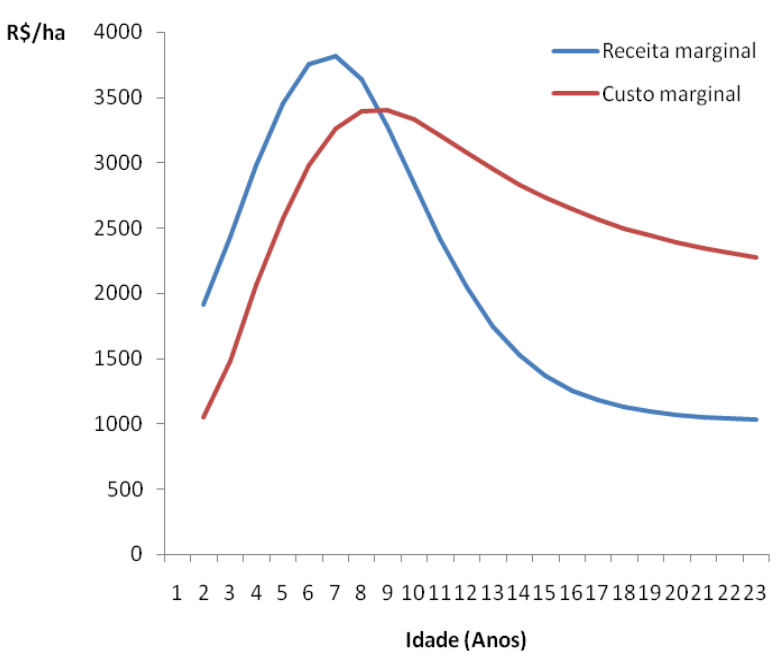

Figura 4 - Idade ótima de corte estipulada pelo método gráfico, considerando-se o preço de venda da cultura agrícola de $\mathrm{R} \$ 240 /$ saca.

Figure 4-Optimal cutting age stipulated by the graphical method, considering the selling price of the crop at $R \$ 240 / \mathrm{bag}$.

antecipar o corte da cultura arbórea; ou seja, é mais vantajoso remover as árvores, executar a reforma e reiniciar o sistema, pois as receitas intermediárias decorrentes da cultura agrícola proporcionarão o aumento do VPL.

\section{CONCLUSÕES}

As equações diferenciais demonstram-se eficientes para a análise econômica de SAF. Esta técnica indicou a viabilidade financeira do modelo hipotético de sistema agroflorestal considerado; e diferente das demais esta a vantagem de poder deteminar a duração do ciclo da cultura arbórea, enfatizando o ótimo econômico do sistema.

Considerando-se como horizonte de planejamento apenas um corte da cultura arbórea, variações na receita da cultura agrícola não afetam a idade ótima de corte. Entretanto, em horizonte de planejamento de vários cortes, variações na receita agrícola implicam em variações inversamente proporcionais na idade ótima de corte. Sendo assim, é mais interessante economicamente antecipar o corte das árvores e reiniciar o sistema, implantando novamente a cultura agrícola, pois a venda desse produto proporcionará aumento no VPL, podendo reduzir os custos de implantação do sistema. 


\section{REFERÊNCIAS}

BINOTI, D. H. B. Estratégias de regulação de florestas equiâneas com vista ao manejo de paisagem. 2010. 145f. Dissertação (Mestrado em Ciência Florestal) Universidade Federal de Viçosa, Viçosa, MG, 2010.

CAMPELLO, E. F. C. et al.. Implantação e manejo de SAF's na Mata Atlântica: a experiência da embrapa agrobiologia. In: Sistemas

Agroflorestais: base científica para o desenvolvimento sustentável. Campos dos Goytacazes: Universidade Estadual do Norte Fluminense Darcy Ribeiro, 2006. p.33-42.

DOSSA, D. A decisão econômica num sistema agroflorestal. Colombo: Embrapa Floresta, 2000. 24p. (Circular Técnica, 39).

DUBÈ, F. et al. Avaliação econômica de um sistema agroflorestal com Eucalyptus sp. no noroeste de Minas Gerais: o caso da Companhia Mineira de Metais. Revista Árvore, v.24, n.4, p.437-443, 2000.

ENGEL, V. L. Introdução aos Sistemas Agroflorestais. Botucatu: FEPAF, 1999. 70 p.

LOPES, H. V. S. Análise econômica dos fatores que afetam a rotação de povoamentos de eucaliptos. Viçosa, MG: Universidade Federal de Viçosa, 1990. 188p.

MARQUES, G. M. et al. Uso de equações diferenciais para determinação do momento ótimo de transformação de veículos. Revista Árvore, v.29, n.1, p.95-104, 2005.

OTS/CATIE. Sistemas Agroforestales: principios y apllicaciones en los tropicos. San Jose: Organización para Estudios Tropicales/ CATIE, 1986. 818p.

PASSOS, C. A. M.; COUTO, L. Sistemas agroflorestais potenciais para o Estado do Mato Grosso do Sul. In: SEMINÁRIO SOBRE SISTEMAS FLORESTAIS PARA O MATO GROSSO DO SUL, 1997, Dourados. Resumos... Dourados: Embrapa-CPAO, 1997. p.16-22 (Documento, 10)
REZENDE, J. L. P.; OLIVEIRA, A. D. Avaliação de projetos florestais. Viçosa, MG: Universidade Florestal de Viçosa, Imprensa Universitária, 1995. 47p.

RIBEIRO, R. N. S.; SANTANA, A. C.; TOURINHO, M. M. Análise exploratória da socioeconomia de sistemas agroflorestais em Várzea Flúvio-Marinha, Cametá-Pará, Brasil.

Revista de Economia e Sociologia Rural, v.42, n.1, p.133-152, 2004.

RIBEIRO, S. C. et al. Estimativa do abatimento de erosão aportado por um sistema agrossilvipastoril e sua contribuição econômica. Revista Árvore, v.31, n.2, p.285-293, 2007.

RODIGHERI, H. R. Rentabilidade econômica comparativa entre plantios florestais e sistemas agroflorestais com erva-mate, eucalipto, pinus e as culturas do feijão, milho, soja e trigo. Colombo: Embrapa-CNPF, 1997. 36p. (Circular Técnica, 26).

SANTOS, A. J. et al. Viabilidade econômica do sistema agroflorestal grevílea x café na Região Norte do Paraná. Revista Cerne, v.6, n.1, p.89-100, 2000.

SANTOS, M. J. C.; PAIVA, S. N. Os sistemas agroflorestais como alternativa econômica em pequenas propriedades rurais: estudo de caso. Ciência Florestal, v.12, n.1, p.135-141, 2002.

SILBERBERG, E.; SUEN, W. The structure of economics: a mathematical analysis. 3.ed. 2000. 668 .

SILVA, M. L.; JACOVINE, L. A. G.; VALVERDE, S. R. Economia florestal. Viçosa, MG: Universidade Federal deViçosa, Imprensa Universitária, 2002. 178p.

SILVA, M. L.; FONTES, A. A. Discussão sobre os critérios de avaliação econômica: Valor Presente Líquido (VPL), Valor Anual Equivalente (VAE) e Valor Esperado da Terra (VET). Revista Árvore, v.29, n.6, p.931-936, 2005.

SOUZA, A. N. et al. Viabilidade econômica de um sistema agroflorestal. Revista Cerne, v.13, n.1, p.96-106, 2007. 
